\title{
A CHARACTERIZATION OF SCREEN CONFORMAL HALF LIGHTLIKE SUBMANIFOLDS
}

\author{
DAe Ho JiN
}

Abstract. We study the geometry of screen conformal half lightlike submanifolds of a semi-Riemannian manifod. The main result is a classification theorem for screen conformal half lightlike submanifolds of a semi-Riemannian space form with a Killing co-screen distribution.

\section{Introduction}

It is well known that the radical distribution $\operatorname{Rad}(T M)=T M \cap$ $T M^{\perp}$ of a half lightlike submanifold $M$ of a semi-Riemannian manifold $(\bar{M}, \bar{g})$ is a vector subbundle of the tangent bundle $T M$ and the normal bundle $T M^{\perp}$ of rank 1. There exist two complementary non-degenerate distributions $S(T M)$ and $\mathcal{D}$ of $\operatorname{Rad}(T M)$ in $T M$ and $T M^{\perp}$ respectively, called a screen and co-screen distribution on $M$ such that

$$
T M=\operatorname{Rad}(T M) \oplus_{\text {orth }} S(T M), T M^{\perp}=\operatorname{Rad}(T M) \oplus_{\text {orth }} \mathcal{D},
$$

where the symbol $\oplus_{\text {orth }}$ denotes the orthogonal direct sum. We denote such a half lightlike submanifold by $(M, g, S(T M))$. Denote by $F(M)$ the algebra of smooth functions on $M$ and by $\Gamma(E)$ the $F(M)$ module of smooth sections of a vector bundle $E$ over $M$. Then there exist vector fields $\xi \in \Gamma(\operatorname{Rad}(T M))$ and $u \in \Gamma(\mathcal{D})$ such that

$$
\bar{g}(u, u)=\epsilon= \pm 1, \quad \bar{g}(\xi, v)=0,{ }^{\forall} v \in \Gamma\left(T M^{\perp}\right) .
$$

Consider the orthogonal complementary distribution $S(T M)^{\perp}$ to $S(T M)$ in $T \bar{M}$. Certainly $\xi$ and $u$ belong to $\Gamma\left(S(T M)^{\perp}\right)$. Hence we have the following orthogonal decomposition

$$
S(T M)^{\perp}=\mathcal{D} \oplus_{\text {orth }} \mathcal{D}^{\perp},
$$

where $\mathcal{D}^{\perp}$ is the orthogonal complementary to $\mathcal{D}$ in $S(T M)^{\perp}$. We known [3] that, for any null section $\xi$ of $\operatorname{Rad}(T M)$ on a neighborhood $\mathcal{U} \subset M$,

Received August 21, 2008. Accepted March 9, 2009.

Key words and phrases: Half lightlike submanifolds, Screen conformals.

This work was supported by Dongguk University Research Fund. 
there exists a uniquely defined vector field $N \in \Gamma(\operatorname{ltr}(T M))$ satisfying

$$
\bar{g}(\xi, N)=1, \bar{g}(N, N)=\bar{g}(N, X)=\bar{g}(N, u)=0,
$$

for all $X \in \Gamma(S(T M) \mid \mathcal{U})$. We call $\operatorname{ltr}(T M), N$ and $\operatorname{tr}(T M)=\mathcal{D} \oplus_{\text {orth }}$ ltr $(T M)$ the lightlike transversal vector bundle, lightlike transversal vector field and transversal vector bundle of $M$ with respect to $S(T M)$ respectively. $T \bar{M}$ is decomposed as follow:

$$
T \bar{M}=T M \oplus \operatorname{tr}(T M) .
$$

The purpose of this paper is to study the geometry of screen conformal half lightlike submanifolds of a semi-Riemannian space form $\bar{M}(c)$. We prove a characterization theorem for $M \operatorname{such}$ that $\operatorname{dim} M>3$ : If the co-screen distribution is Killing, then $c=0$ (Theorem 2.1). Using this theorem, we prove an annexed theorem: If $c \neq 0$, then $M$ is totally umbilical and locally a product manifold $M=C \times M^{*}$, where $C$ is a null curve and $M^{*}$ is a totally umbilical semi-Riemannian 2-surface or a non-null curve (Theorem 2.3). Recall the following structure equations:

Let $\bar{\nabla}$ be the Levi-Civita connection of $\bar{M}$ and $P$ the projection morphism of $\Gamma(T M)$ on $\Gamma(S(T M))$ with respect to the decomposition (1.1). Then the local Gauss and Weingartan formulas are given by

$$
\begin{aligned}
& \bar{\nabla}_{X} Y=\nabla_{X} Y+B(X, Y) N+D(X, Y) u, \\
& \bar{\nabla}_{X} N=-A_{N} X+\tau(X) N+\rho(X) u \\
& \bar{\nabla}_{X} u=-A_{u} X+\phi(X) N \\
& \nabla_{X} P Y=\nabla_{X}^{*} P Y+C(X, P Y) \xi \\
& \nabla_{X} \xi=-A_{\xi}^{*} X-\tau(X) \xi
\end{aligned}
$$

for any $X, Y \in \Gamma(T M)$, where $\nabla$ and $\nabla^{*}$ are induced linear connections of $M$ and on $S(T M)$ respectively, the bilinear forms $B$ and $D$ on $T M$ are called the local transversal second fundamental form and local screen second fundamental form of $M$ respectively, $C$ is called the local radical second fundamental form on $S(T M) . A_{N}, A_{\xi}^{*}$ and $A_{u}$ are linear operators on $\Gamma(T M)$ and $\tau, \rho$ and $\phi$ are 1-forms on $T M$.

Since the connection $\bar{\nabla}$ of $\bar{M}$ is torsion-free, the induced connection $\nabla$ of $M$ is also torsion-free and both $B$ and $D$ are symmetric and

$$
B(X, \xi)=0, \quad D(X, \xi)=-\epsilon \phi(X),{ }^{\forall} X \in \Gamma(T M) .
$$

The induced connection $\nabla$ on $M$ is not metric and satisfies

$$
\left(\nabla_{X} g\right)(Y, Z)=B(X, Y) \eta(Z)+B(X, Z) \eta(Y),
$$


for all $X, Y, Z \in \Gamma(T M)$, where $\eta$ is a 1 -form on $T M$ such that

$$
\eta(X)=\bar{g}(X, N),{ }^{\forall} X \in \Gamma(T M) .
$$

But the connection $\nabla^{*}$ on $M^{*}$ is metric. The above three local second fundamental forms of $M$ and $M^{*}$ are related to their shape operators by

$$
\begin{aligned}
& B(X, Y)=g\left(A_{\xi}^{*} X, Y\right), \quad \bar{g}\left(A_{\xi}^{*} X, N\right)=0, \\
& C(X, P Y)=g\left(A_{N} X, P Y\right), \quad \bar{g}\left(A_{N} X, N\right)=0, \\
& \epsilon D(X, P Y)=g\left(A_{u} X, P Y\right), \quad \bar{g}\left(A_{u} X, N\right)=\epsilon \rho(X), \\
& \epsilon D(X, Y)=g\left(A_{u} X, Y\right)-\phi(X) \eta(Y) .
\end{aligned}
$$

We show, by (1.12) and (1.13), that $A_{\xi}^{*}$ and $A_{N}$ are $\Gamma(S(T M))$-valued and shape operators related to $B$ and $C$ respectively, called the radical and transversal shape operator on $T M$ and $S(T M)$ respectively. From (1.12), $A_{\xi}^{*}$ is self-adjoint on $\Gamma(T M)$ such that $A_{\xi}^{*} \xi=0$.

A vector field $X$ on a semi-Riemannian manifold $(\bar{M}, \bar{g})$ is said to be a Killing if $\overline{\mathcal{L}}_{X} \bar{g}=0$, where the symbol $\overline{\mathcal{L}}_{X}$ denotes the Lie derivative with respect to the vector field $X$. A distribution $D$ on $\bar{M}$ is said to be a Killing if each vector field belonging to $D$ is a Killing. If the co-screen distribution $\mathcal{D}$ is a Killing, using (1.6) and (1.15), we have

$$
\left(\overline{\mathcal{L}}_{u} \bar{g}\right)(X, Y)=-2 \epsilon D(X, Y) .
$$

Theorem 1.1 [5]. Let $(M, g, S(T M))$ be a half lightlike submanifold of a semi-Riemannian manifold $(\bar{M}, \bar{g})$. Then the co-screen distribution is a Killing distribution if and only if $D=0$ on $\Gamma(T M)$.

We denote by $\bar{R}, R$ and $R^{*}$ the curvature tensors of $\bar{\nabla}, \nabla$ and $\nabla^{*}$ respectively. Using the Gauss-Weingarten equations for $M$ and $S(T M)$, we obtain the Gauss-Codazzi equations for $M$ and $S(T M)$ :

$$
\begin{gathered}
\bar{g}(\bar{R}(X, Y) Z, P W)=g(R(X, Y) Z, P W) \\
+B(X, Z) C(Y, P W)-B(Y, Z) C(X, P W) \\
+\epsilon\{D(X, Z) D(Y, P W)-D(Y, Z) D(X, P W)\} \\
\bar{g}(\bar{R}(X, Y) Z, \xi)=\left(\nabla_{X} B\right)(Y, Z)-\left(\nabla_{Y} B\right)(X, Z) \\
+B(Y, Z) \tau(X)-B(X, Z) \tau(Y) \\
\quad+D(Y, Z) \phi(X)-D(X, Z) \phi(Y) \\
\bar{g}(\bar{R}(X, Y) Z, N)=\bar{g}(R(X, Y) Z, N) \\
+\epsilon\{D(X, Z) \rho(Y)-D(Y, Z) \rho(X)\}
\end{gathered}
$$




$$
\begin{gathered}
\bar{g}(R(X, Y) P Z, P W)=g\left(R^{*}(X, Y) P Z, P W\right) \\
+C(X, P Z) B(Y, P W)-C(Y, P Z) B(X, P W), \\
g(R(X, Y) P Z, N)=\left(\nabla_{X} C\right)(Y, P Z)-\left(\nabla_{Y} C\right)(X, P Z) \\
+C(X, P Z) \tau(Y)-C(Y, P Z) \tau(X) .
\end{gathered}
$$

\section{Screen conformal half lightlike submanifolds}

A half lightlike submanifold $(M, g, S(T M))$ of a semi-Riemannian manifold $(\bar{M}, \bar{g})$ is screen conformal [1] if the shape operators $A_{N}$ and $A_{\xi}^{*}$ of $M$ and $S(T M)$ respectively are related by $A_{N}=\varphi A_{\xi}^{*}$, or equivalently,

$$
C(X, P Y)=\varphi B(X, Y),{ }^{\forall} X, Y \in \Gamma(T M)
$$

where $\varphi$ is a non-vanishing smooth function on a neighborhood $\mathcal{U}$ in $M$.

Note. For a screen conformal half lightlike submanifold $M$, as $C$ is symmetric, $S(T M)$ is integrable. Thus $M$ is a locally product manifold $C \times M^{*}$ where $C$ is a null curve and $M^{*}$ is a leaf of $S(T M)$ [3].

Theorem 2.1. Let $(M, g, S(T M))$ be an $m(>3)$-dimensional screen conformal half lightlike submanifold of a semi-Riemannian space form $\bar{M}(c)$ with a Killing co-screen distribution. Then $c=0$.

Proof. As $\bar{M}$ is a space of constant curvature with a Killing co-screen distribution, by Theorem 1.1, (1.9), (1.14) and (1.15), we have

$$
D(X, Y)=0, \quad \phi(X)=0, \quad A_{u} X=\epsilon \rho(X) \xi,
$$

for any $X, Y \in \Gamma(T M)$. From (1.17), we obtain

$$
\left(\nabla_{X} B\right)(Y, Z)-\left(\nabla_{Y} B\right)(X, Z)=B(X, Z) \tau(Y)-B(Y, Z) \tau(X)
$$

for all $X, Y, Z \in \Gamma(T M)$. Using this, (1.18), (1.20) and (2.1), we get

$$
\begin{gathered}
\{X[\varphi]-2 \varphi \tau(X)\} B(Y, P Z)-\{Y[\varphi]-2 \varphi \tau(Y)\} B(X, P Z) \\
=c\{g(Y, P Z) \eta(X)-g(X, P Z) \eta(Y)\} .
\end{gathered}
$$

Replacing $Y$ by $\xi$ in (2.3) and using (1.9), we obtain

$$
\{\xi[\varphi]-2 \varphi \tau(\xi)\} B(X, P Z)=c g(X, P Z) .
$$

If $\xi[\varphi]-2 \varphi \tau(\xi)=0$ or $B=0$, i.e., $M$ is a totally geodesic, then $c=0$. 
Assume that $c \neq 0$. Then $\xi[\varphi]-2 \varphi \tau(\xi) \neq 0$ and $B \neq 0$, that is, $M$ is not a totally geodesic. From (2.1) and (2.4), we have

$$
B(X, Y)=\sigma g(X, Y), C(X, Y)=\varphi \sigma g(X, Y),
$$

for all $X, Y \in \Gamma(T M)$, where $\sigma=c(\xi[\varphi]-2 \varphi \tau(\xi))^{-1} \neq 0$. If $\varphi \sigma=0$, then $C=0$ by (2.5). From (2.1), we have $B=0$. Therefore, we get $\varphi \sigma \neq 0$. Thus $M$ and $S(T M)$ are totally umbilical which are not totally geodesic. Since $M$ is screen conformal, by Note, $M$ is a locally product manifold $C \times M^{*}$ where $C$ is a null curve and $M^{*}$ is a leaf of $S(T M)$. Since $\bar{M}$ has a constant curvature, from (1.16), (1.19) and (2.5), we have

$$
R^{*}(X, Y) Z=\left(c+2 \varphi \sigma^{2}\right)\{g(Y, Z) X-g(X, Z) Y\},
$$

for all $X, Y, Z \in \Gamma(S(T M))$. Thus the leaf $M^{*}$ of $S(T M)$ is a semiRiemannian manifold of curvature $\left(c+2 \varphi \sigma^{2}\right)$. Let $R i c^{*}$ be the induced symmetric Ricci tensor of $M^{*}$. From (2.6), we have

$$
\operatorname{Ric}^{*}(X, Y)=\left(c+2 \varphi \sigma^{2}\right)(m-2) g(X, Y),{ }^{\forall} X, Y \in \Gamma(S(T M)) .
$$

Thus $M^{*}$ is an Einstein manifold. Since $\operatorname{dim} M^{*}>2,\left(c+2 \varphi \sigma^{2}\right)$ is a constant and $M^{*}$ has a constant curvature $\left(c+2 \varphi \sigma^{2}\right)$.

Using (1.10), (2.2), (2.5) and the degeneracy of $S(T M)$, we have

$$
\left\{X[\sigma]+\sigma \tau(X)-\sigma^{2} \eta(X)\right\} P Y=\left\{Y[\sigma]+\sigma \tau(Y)-\sigma^{2} \eta(Y)\right\} P X
$$

Now suppose there exists a vector field $X_{o} \in \Gamma(T M)$ such that $X_{o}[\sigma]+$ $\sigma \tau\left(X_{o}\right)-\sigma^{2} \eta\left(X_{o}\right) \neq 0$ at each point $x \in M$, then $P Y=f P X_{o}$ for all $Y \in \Gamma(T M)$, where $f$ is a smooth function. It follows that all vectors from the fiber $S(T M)_{x}$ are co-linear with $\left(P X_{o}\right)_{x}$. It is a contradiction as $\operatorname{dim}\left(S(T M)_{x}\right)>2$. Thus $X[\sigma]+\sigma \tau(X)-\sigma^{2} \eta(X)=0$ for all $X \in$ $\Gamma(T M)$. This implies $\xi[\sigma]=\sigma^{2}-\sigma \tau(\xi)$. Therefore, $0=\xi\left[\varphi \sigma^{2}\right]=\sigma(c+$ $\left.2 \varphi \sigma^{2}\right)$. Since $\left(c+2 \varphi \sigma^{2}\right)$ is a constant and $\sigma \neq 0$, we have $c+2 \varphi \sigma^{2}=0$. Thus $M^{*}$ is a semi-Euclidean space and the second fundamental form $C$ of $M^{*}$ satisfies $C=0$. Thus, from (2.5), we have $\varphi \sigma=0$. This means $c=0$. It is contradiction to $c \neq 0$. Consequently, we have $c=0$.

Corollary 1. There exist no screen conformal half lightlike submanifolds of semi-Riemannian space form $\bar{M}(c), c \neq 0$ with a Killing co-screen distribution and $\operatorname{dim} M>3$.

Corollary 2. There exist no screen conformal totally geodesic half lightlike submanifolds of semi-Riemannian space form $\bar{M}(c), c \neq 0$ with a Killing co-screen distribution. 
Theorem 2.2. Let $(M, g, S(T M))$ be an $m(>3)$-dimensional screen conformal half lightlike submanifold of $\bar{M}(c)$ with a Killing co-screen distribution. Then the curvature tensors $R$ and $R^{*}$ of $M$ and $S(T M)$ respectively, are related by

$$
R(X, Y) Z=\frac{1}{2} R^{*}(P X, P Y) P Z, \quad{ }^{\forall} X, Y, Z \in \Gamma(T M) .
$$

Proof. Form (1.18) with $D=c=0$, we have $\bar{g}(R(X, Y) Z, N)=0$. Thus we see that $(2.9)$ is equivalent with

$$
g(R(X, Y) Z, P W)=\frac{1}{2} g\left(R^{*}(P X, P Y) P Z, P W\right),
$$

for all $X, Y, Z, W \in \Gamma(T M)$. Due to (1.17) with $D=c=0$, we have $g(R(X, Y) \xi, Z)=0$. Thus we see that (2.10) is true for $Z=\xi$. Using (1.9), (1.16) and (1.19) satisfy $D=c=0$ and (2.1), we derive (2.10).

Theorem 2.3. Let $(M, g, S(T M))$ be a screen conformal half lightlike submanifold of a semi-Riemannian space form $\bar{M}(c), c \neq 0$ with a Killing co-screen distribution. Then $M$ is totally umbilical and locally a product manifold $C \times M^{*}$, where $C$ is a null curve and $M^{*}$ is a totally umbilical semi-Riemannian 2-surface or a non-null curve.

Proof. From the fact that $M$ is a screen conformal lightlike hypersurface with $c \neq 0$, we show that $\operatorname{dim} M \leq 3$ and both $M$ and $M^{*}$ are totally umbilical which are not totally geodesic by (2.5). By Note, we get $M=C \times M^{*}$ and $M^{*}$ is a proper totally umbilical semi-Riemannian 2 -surface or a non-null curve.

\section{References}

[1] Atindogbe, C. and Duggal, K. L. Conformal screen on lightlike hypersurfaces, International J. of Pure and Applied Math., 11(4), 2004, 421-442.

[2] Duggal, K. L., On canonical screen for lightlike submanifolds of codimension two, Central European Joural of Math., vol. 5(4), 2007, 710-719.

[3] Duggal, K. L. and Bejancu, A., Lightlike Submanifolds of Semi-Riemannian Manifolds and Applications, Kluwer Acad. Publishers, Dordrecht, 1996.

[4] Jin, D. H., Einstein half lightlike submanifolds of codimension 2, to appear in J. Korea Soc. Math. Educ. Ser. B: Pure Appl. Math., 16, no.1, 2009.

[5] Jin, D. H., Einstein half lightlike submanifolds with a Killing co-screen distribution, Honam Math. J., 30, no.3 2008, p487-504. 
Dae Ho Jin

Department of Mathematics,

Dongguk University,

Gyeongju 780-714, Republic of Korea

E-mail: jindh@dongguk.ac.kr 